\title{
Sensor coverage with a multi-robot system
}

I AP $\mathbf{v}_{\mathbf{2} 22}$ systems \& control

\section{Jonathan A. Rogge, Dirk Aeyels}

SYSTeMS Research Group, EESA Department

Faculty of Engineering, Ghent University Technologiepark-Zwijnaarde 914, 9052 Zwijnaarde

\{jonathan.rogge, dirk. aeyels \} auGent.be

\section{Sensor coverage}

Two mission types:

- Static targets: mine field clearance, search and rescue missions

Moving targets: intercept missions

Given:

- Fixed area $S$, containing disjoint convex obstacles and targets

- The obstacles are not located on the boundary of $S$.

- Location, size and shape of the obstacles are unknown.

Goal: The robot sensors

- cover the entire free space in order to

- locate(=sense) all static targets and/or

- intercept(=sense) all moving targets.

\section{Restrictions}

- All robots possess a compass to determine their orientation

- Only the outer robots possess a GPS system to determine their position

- The robots have no knowledge about the terrain

- They have restricted memory capabilities

- They do not perform map making or path planning

- Communication between robots has a finite range and is only possible if the line-of-sight is not obstructed.

- A limited set of commands/properties is communicated between robots:

- assignment of leader and follower,

- status red, green, or orange

- P2O-value left, right, or empty

\section{Leader-follower structure}

- Each robot $i$ is at a point in 2D-space, with coordinates $\left(x_{i}, y_{i}\right)$ - It checks two locations in its surroundings for occupation by other robots, namely

- LNP = left neighbour position $\left(x_{i}-d, y_{i}\right)$

$-\mathrm{RNP}=$ left neighbour position $\left(x_{i}+d, y_{i}\right)$

- The robot at LNP is the leader of the robot $i$; the robot at RNP is its follower

$\Rightarrow$ Creation of a leader-follower structure of the entire robot group.

The leftmost robot has no leader and can be regarded as the leader of the entire group. The rightmost robot has no follower. N.B. relative measurements between robots and a fixed direcN.B. relative measurements between robots and a fixed direc-
tion obtained by a compass suffice to determine LNP and RNP.

\section{Algorithm description: preliminaries}

- Each robot divides its surrounding space into 4 quadrants. - $\mathrm{P} 2 \mathrm{O}=$ position of the robot w.r.t. an obstacle (left, right, or empty)

- Dist2O = shortest distance between robot and an obstacle

Each robot takes on a status:

- Status Green: Advance along a fixed direction maintaining your leader at LNP

- Code Orange: Move clockwise around obstacle

- Code Red: Stand still

The robots scan the area $S$ in one single sweep.

Changes in the robot's sensed surroundings or commands re

ceived from other robots induce changes of status in a robot.

We explain these triggers and switches using a simulation ex

ample.
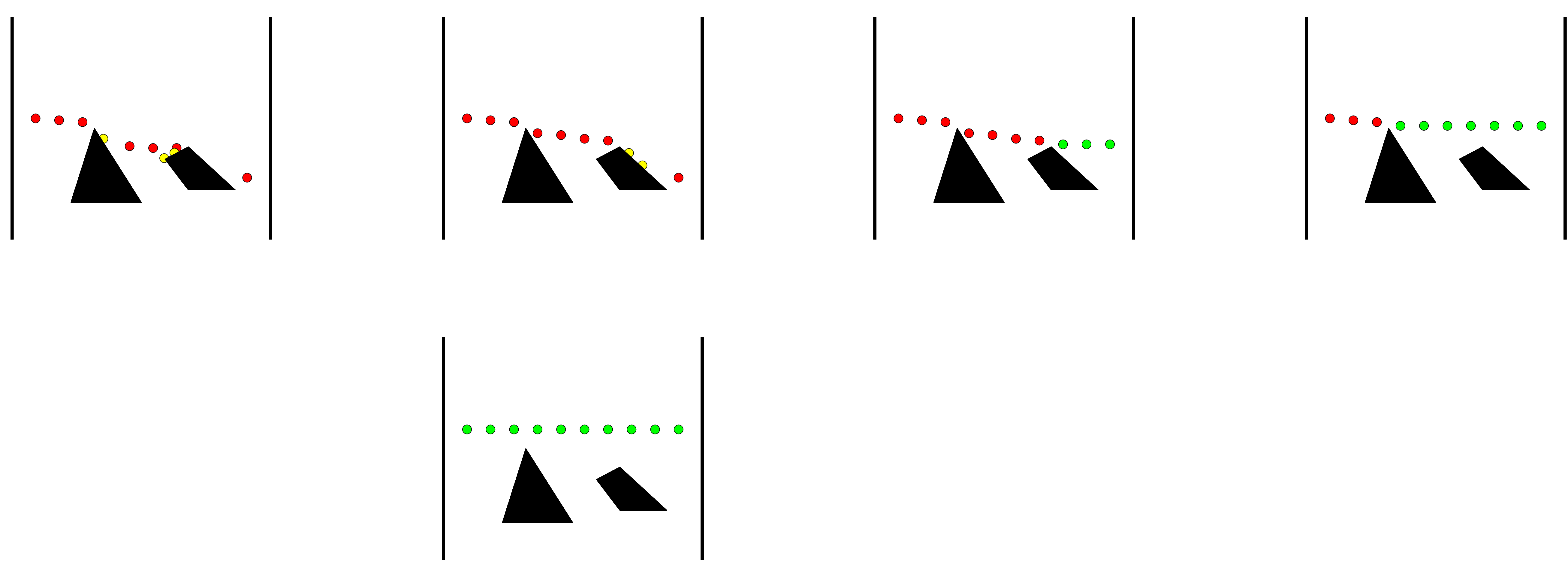